\title{
DEPENDENCE OF ARITHMETIC FUNCTIONS AND DIRICHLET SERIES
}

\author{
VICHIAN LAOHAKOSOL
}

(Communicated by William W. Adams)

\begin{abstract}
Simpler proofs of results about algebraic dependence are given in the domain of arithmetic functions under addition and convolution and in the domain of Dirichlet series. Better measures of differential transcendence are derived in the latter case.
\end{abstract}

\section{INTRODUCTION}

By arithmetic functions we mean complex-valued functions whose domain is the set of positive integers. The convolution (or Dirichlet product) of two arithmetic functions $f$ and $g$ are defined by

$$
(f * g)(n)=\sum_{i j=n} f(i) g(j) .
$$

For brevity, we write $f^{* k}$ for $f * f * \cdots * f$ ( $k$ times). It is well known that $\mathscr{A}$, the set of all arithmetic functions, is an integral domain under addition and convolution $[1,4,8]$. The domain $\mathscr{A}$ has two other representations: one as the domain of formal Dirichlet series and the other as the domain of formal power series in a countable set of indeterminates $[4,11,12]$. The function $u$, defined by $u(n)=1$ if $n=1$ and $u(n)=0$ otherwise, plays the role of the identity with respect to $*$. Following [8], we define the $k$ th zeta function by

$$
\zeta_{k}(n)=n^{k} \text {. }
$$

Many well-known arithmetic functions are known to be related to different zeta functions $[3,8]$, e.g.,

$$
\mu * \zeta_{0}=u, \quad d=\zeta_{0}^{* 2}, \quad \sigma=\zeta_{0} * \zeta_{1}, \quad \phi=\zeta_{1} * \mu=\zeta_{1} * \zeta_{0}^{*-1},
$$

where $\mu$ is the Möbius function, $d(n)$ the number of divisors of $n, \sigma(n)$ the sum of the divisors of $n$, and $\phi$ the Euler function. A set of arithmetic functions $f_{1}, \ldots, f_{r}$ is said to be (ordinarily) algebraically dependent if there exists a nontrivial polynomial $P\left(X_{1}, \ldots, X_{r}\right)$ with complex coefficients such that

Received by the editors December 7, 1990.

1991 Mathematics Subject Classification. Primary 11J85, 11A25, 11 J91.

The author's research was partially supported by a grant from Kasetsart University, Bangkok, Thailand. 
$P\left(f_{1}, \ldots, f_{r}\right)=0$. This kind of dependence was first investigated by Bellman and Shapiro [2]. They showed that $\zeta_{1}, \phi, d, \sigma, \mu$, and $2^{\nu}$ are algebraically independent, where $\nu(n)$ is the number of distinct primes dividing $n$; see also [18] for a short proof of a smaller case. Because of its application to two other representations, it is more natural to consider not ordinary algebraic dependence, but $*$-algebraic dependence that is defined as follows: a set of arithmetic functions $f_{1}, \ldots, f_{r}$ is said to be *-algebraically dependent if there exists a nontrivial polynomial $P\left(X_{1}, \ldots, X_{r}\right):=\sum_{(i)} a_{(i)} X_{1}^{i_{1}} \cdots X_{r}^{i_{r}}$ with complex coefficients such that $P\left(f_{1}, \ldots, f_{r}\right):=\sum_{(i)} a_{(i)} f_{1}^{* i_{1}} \ldots f_{r}^{* i_{r}}=0$ and is said to be *-algebraically independent otherwise. The first investigation of *-algebraic dependence appeared in [3]. There it was shown by elementary means that for nonnegative integers $r$ and $s$, the functions $\zeta_{0}, \ldots, \zeta_{r}, Q_{0}, \ldots, Q_{s}$ are *algebraically independent, where $Q_{k}(n)=n^{k}$ if $n$ is squarefree and $Q_{k}(n)=0$ otherwise. Popken [11] considered *-algebraic dependence in a more general setting of functions defined over a unique factorization semigroup with values in a ring. His main results give necessary conditions for *-algebraic dependence. This was done by analyzing the Taylor expansion of the polynomial representing dependence. In subsequent papers $[13,14]$ he made applications to Dirichlet series and multiplicative arithmetic functions.

In the direction of Dirichlet series, Popken [15] also gave a measure for the so-called differential transcendence of certain Dirichlet series closely connected to the Riemann zeta function. More recent works are due to Shapiro [16] and Shapiro and Sparer [17]. In [16] a number of basic important notions such as derivation and valuation were developed, while in [17] the authors considered, among other things, algebraic independence of Dirichlet series and transcendence over $\mathbb{C}[\zeta]$, where $\zeta$ is the Riemann zeta function.

In this paper we give a simpler proof of $*$-algebraic dependence criteria, due to Popken [11], which actually reveals systematically how to derive more refined conditions. We also obtain measures for differential transcendence of certain Dirichlet series superseding those established by Popken [15]. The improvements arise from the use of derivations as expounded in [16].

A derivation $d$ over $\mathscr{A}$ is a mapping of $\mathscr{A}$ into itself satisfying $d(f * g)=d f * g+f * d g, d\left(c_{1} f+c_{2} g\right)=c_{1} d f+c_{2} d g$ for all $f, g$ in $\mathscr{A}$ and complex constants $c_{1}, c_{2}$ [16]. Two typical examples of derivation that will be repeatedly used are

(i) $\log$-derivation $\left(d_{1} f\right)(n)=f(n) \log n$;

(ii) basic (or p-basic) derivation, $p$ rational prime, $\left(d_{p} f\right)(n)=f(n p) v_{p}(n p)$, where $v_{p}(n)$ denotes the exponent of the highest power of $p$ dividing $n$.

\section{2. *-ALGEBRAIC DEPENDENCE}

Results concerning *-algebraic dependence have been obtained by many authors $[3,6,7,11,12,17]$. Our objective in this section is to illustrate the power and use of derivations by offering a much simpler and more revealing proof of conditions for *-algebraic dependence first established by Popken [11].

Theorem 1. If the arithmetic functions $f_{1}, \ldots, f_{r}$ are *-algebraically dependent then there exist complex numbers $c_{i}, c_{i j}(1 \leq i \leq j \leq r)$, not all 0 , such that 
the relations,

$$
\begin{aligned}
& \sum_{i=1}^{r} c_{i} f_{i}(p)=0 \\
& \sum_{i=1}^{r} c_{i} f_{i}\left(p^{2}\right)+\sum_{1 \leq i \leq j \leq r} c_{i j} f_{i}(p) f_{j}(p)=0 \\
& \sum_{i=1}^{r} c_{i} f_{i}(p q)+\sum_{1 \leq i \leq j \leq r} c_{i j}\left(f_{i}(p) f_{j}(q)+f_{i}(q) f_{j}(p)\right)=0
\end{aligned}
$$

hold simultaneously for all primes $p, q(\neq p)$ except finitely many.

Proof. Consider a polynomial (in $1, \ldots, f_{r}$ ) with complex coefficients

$$
Q(n)=\sum_{(i)} a_{(i)} f_{1}^{* i_{1}} * \cdots * f_{r}^{* i_{r}}(n) .
$$

Taking $p$-basic derivation, we get

$$
d_{p} Q(n)=\sum_{j=1}^{r} Q_{j} * d_{p} f_{j}(n)
$$

where

$$
Q_{j}\left(:=\frac{\partial Q}{\partial f_{j}}\right)=\sum_{(i)} a_{(i)} i_{j} f_{1}^{* i_{1}} * \cdots * f_{j}^{* i_{j}-1} * \cdots * f_{r}^{* i_{r}} .
$$

Expanding out, we have

$$
Q(p n) v_{p}(p n)=\sum_{j=1}^{r} \sum_{s t=n} Q_{j}(s) f_{j}(p t) v_{p}(p t)
$$

If $f_{1}, \ldots, f_{r}$ are $*$-algebraically dependent, then there exists such a nontrivial polynomial $Q$ vanishing for all $n$. We may assume that $Q$ has the least total degree. Since $Q_{j}$ is a polynomial of lower total degree, then there exists a positive integer $n_{0}$ such that $Q_{j}\left(n_{0}\right) \neq 0$ for some $j$, while $Q_{j}(n)=0$ for all $j$ and $n<n_{0}$. Putting $n=n_{0}$ into (2.1) and using $*$-algebraic dependence, we arrive at

$$
0=Q\left(p n_{0}\right) v_{p}\left(p n_{0}\right)=\sum_{j=1}^{r} Q_{j}\left(n_{0}\right) f_{j}(p),
$$

which is the first assertion. Taking another $p$-basic derivation in (2.0), we get

$$
d_{p}^{2} Q(n)=\sum_{j=1}^{r} Q_{j} * d_{p}^{2} f_{j}(n)+\sum_{j=1}^{r} \sum_{k=1}^{r} Q_{j k} * d_{p} f_{j} * d_{p} f_{k}(n)
$$

where

$$
d_{p} Q_{j}=\sum_{k=1}^{r} Q_{j k} * d_{p} f_{k}
$$

Let $n_{1}$ be a positive integer such that $Q_{j}(n)=Q_{j k}(n)=0$ for $n<n_{1}$ and all $j, k$, while $Q_{j}\left(n_{1}\right) \neq 0$ for some $j$ or $Q_{j k}\left(n_{1}\right) \neq 0$ for some $j, k$. Expanding 
and putting $n=n_{1}$ in (2.2) leads to

$$
0=\sum_{j=1}^{r} 2 Q_{j}\left(n_{1}\right) f_{j}\left(p^{2}\right)+\sum_{j, k} Q_{j k}\left(n_{1}\right) f_{j}(p) f_{k}(p),
$$

which is the second assertion of the theorem. On the other hand, if we take $q$-basic derivation $(q \neq p)$ of $(2.0)$ and proceed in the same manner, we get the third assertion and this completes the proof.

Remarks. (i) It becomes clear from the proof of the theorem how higher order conditions for *-algebraic dependence can be derived. Note also the ease of deriving these conditions here in contrast to the equating of Taylor coefficients in [11].

(ii) Through isomorphism, it follows from Theorem 1 that the Riemann zeta function does not satisfy an algebraic differential-difference equation. This is a classical result of Ostrowski [9], referred to several times by Popken [11, 13, 14].

\section{DIRICHLET SERIES}

A (formal) Dirichlet series is a series of the form $D(s)=\sum_{n=1}^{\infty} f(n) / n^{s}$ where $f(n)$ are complex numbers. As mentioned in $\S 1$ the totality of such Dirichlet series, $\mathscr{D}$, is isomorphic to $\mathscr{A}$ and hence is an integral domain with respect to addition and usual multiplication. The log-derivation in $\mathscr{A}$ corresponds to the usual differentiation with respect to $s$ in $\mathscr{D}$, and we use the same notation. Also, with no confusion, both in $\mathscr{D}$ and in $\mathscr{A}$, we employ the same notation for $p$-basic derivation, i.e.,

$$
d_{p} D(s)=\sum_{n \geq 1} d_{p} f(n) / n^{s}
$$

$p$-basic derivation is indeed a derivation in $\mathscr{D}$ as can be directly checked. A Dirichlet series is termed differentially algebraic if it satisfies a nontrivial algebraic differential equation with complex coefficients and is termed differentially transcendental otherwise; here differential refers to the log-derivation, or, what is the same thing, differentiation with respect to $s$. Our main concerns now are to make quantitative the following two results:

(i) the Dirichlet series with nonzero $p$ th coefficients, for infinitely many primes $p$, are differentially transcendental (Ostrowski [9]); this of course includes the case of Riemann zeta function;

(ii) the Riemann zeta function does not satisfy a nontrivial algebraic differential-difference equation.

Such quantitative results are provided through the use of measure of differential transcendence, which is defined to be the valuation of an algebraic expression representing differential or differential-difference algebraicity.

Let $f$ be in $\mathscr{A}$. The valuation of $f$ is defined to be (Shapiro [16], Popken [15]) $|f|=1 / n_{0}$ where $n_{0}$ is the smallest positive integer such that $f\left(n_{0}\right) \neq 0$. Correspondingly, for Dirichlet series $D(s)=\sum f(n) / n^{s}$, its valuation is defined to be the same value, i.e., $|D|=|f|$. It is readily checked that this is a valid valuation in the two domains. Indeed, both valuations are non-archimedean.

We now proceed by proving an auxiliary result that renders more precise information than Lemma 1 of Popken [15]. 
Theorem 2. Let $P\left(X_{0}, \ldots, X_{r}\right)$ be a nontrivial polynomial with complex coefficients and let $D(s)=\sum f(n) / n^{s}$ be in $\mathscr{D}$. Formally, put as an element in $\mathscr{D}$,

$$
P\left(D, D^{\prime}, \ldots, D^{(r)}\right):=\sum F(n) / n^{s},
$$

and

$$
P_{j}\left(D, D^{\prime}, \ldots, D^{(r)}\right):=\frac{\partial P}{\partial X_{j}}\left(D, D^{\prime}, \ldots, D^{(r)}\right):=\sum F_{j}(n) / n^{s} .
$$

Then for any positive intger $n$ and any prime $p$, we have

$$
F(p n) v(p n)=\sum_{j=0}^{r} \sum_{k \mid n} f(p k) v(p k)(-\log p k)^{j} F_{j}(n / k)
$$

and

$$
F(n) \log n=\sum_{j=0}^{r} \sum_{k \mid n} f(k)(-\log k)^{j+1} F_{j}(n / k),
$$

where $v(n)=v_{p}(n)$ is the exponent of the highest power of $p$ dividing $n$. Proof. For prime $p$, let $d:=d_{p}$ be its $p$-basic derivation. Then

$$
d P\left(D, D^{\prime}, \ldots, D^{(r)}\right)=\sum_{j=0}^{r} d D^{(j)} \frac{\partial P}{\partial X_{j}}\left(D, D^{\prime}, \ldots, D^{(r)}\right),
$$

i.e.,

$$
\sum_{n \geq 1} F(p n) v(p n) / n^{s}=\sum_{j=0}^{r}\left(\sum_{n \geq 1} f(p n)(-\log p n)^{j} v(p n) / n^{s}\right)\left(\sum_{n \geq 1} F_{j}(n) / n^{s}\right) .
$$

The first assertion of the theorem follows by interchanging the order of summation of the right-hand side and equating the coefficients of $n^{-s}$. The second assertion is obtained analogously using log-derivation.

Theorem 3. Let $D(s)=\sum f(n) / n^{s}$ be in $\mathscr{D}$, and let $P\left(X_{0}, \ldots, X_{r}\right)$ be a nontrivial polynomial with complex coefficients and of total degree $g$. If $f(p) \neq 0$ for each prime $p$, then there exists a positive, absolute, and computable constant $c$ such that

$$
\left|P\left(D, D^{\prime}, \ldots, D^{(r)}\right)\right| \geq \begin{cases}\{c(r+1) \log (r+1)\}^{-g} & \text { if } r \geq 1, \\ 2^{-g} & \text { if } r=0 .\end{cases}
$$

Proof. If $\operatorname{deg} P=0$, then clearly $\left|P\left(D, D^{\prime}, \ldots, D^{(r)}\right)\right|=1$. If $\operatorname{deg} P=1$, then we can write

$$
P\left(X_{0}, \ldots, X_{r}\right)=a+\sum_{j=0}^{r} a_{j} X_{j},
$$

with the coefficients $a_{j}$ not all vanishing simultaneously. Using the notation of Theorem 2, we see by equating coefficients that for $n \geq 2$,

$$
F(n)=f(n) \sum_{j=0}^{r} a_{j}(-\log n)^{j} .
$$


Since the polynomial $\sum_{j=0}^{r} a_{j} t^{j}$ has at most $r$ zeros and $f(p) \neq 0$ for each prime $p$, it follows that among the values at the first $r+1$ primes, $F\left(p_{1}\right), \ldots$, $F\left(p_{r+1}\right)$, at least one must be nonzero. Therefore, $\left|P\left(D, D^{\prime}, \ldots, D^{(r)}\right)\right| \geq p_{r+1}^{-1}$. By Chebychev's inequality (Apostol [1]), there exists a positive, absolute, and computable constant $c$ such that $p_{r} \leq c r \log r$. Substituting this estimate, we get the theorem in this case.

We now proceed by induction on $\operatorname{deg} P$. Let $P$ be of total degree $g+1 \geq 2$, and assume that the assertion has already been proved for polynomials of total degree $\leq g$. Consequently, unless $P_{j}:=\partial P / \partial X_{j}$ vanishes identically, we must have by induction

$$
\left|P_{j}\left(D, D^{\prime}, \ldots, D^{(r)}\right)\right| \geq B^{-1}, \quad \text { where } B=(c(r+1) \log (r+1))^{g} .
$$

Using the terminology of Theorem 2 , we have that not all of the $[B]$ vectors

$$
\left(F_{j}(1)\right)_{j=0}^{r}, \ldots,\left(F_{j}([B])\right)_{j=0}^{r}
$$

can be zero vectors. Let $\left(F_{j}(m)\right)_{j=0}^{r}(1 \leq m \leq[B])$ be the first nonzero vector in this sequence so that

$$
F_{j}(d)=0 \text { for } 1 \leq d \leq m-1,0 \leq j \leq r .
$$

Using the first assertion of Theorem 2 and the minimality of $m$, we get

$$
F(p m) v(p m)=f(p) \sum_{j=0}^{r} a_{j}(m)(\log p)^{j},
$$

where $a_{j}(m)=(-1)^{j} F_{j}(m)$, with at least one $a_{j}(m) \neq 0$. It follows by the same arguments as in the previous case that among the $r+1$ values $F\left(p_{1} m\right), \ldots$, $F\left(p_{r+1} m\right)$ (we drop the $v(p m)$-factors for they are never 0 ), at least one must be nonzero, i.e., there exists a positive integer $N$ of the form $\mathrm{pm}$ such that $F(N) \neq 0$ and, by Chebychev's inequality,

$$
1 \leq N \leq p_{r+1}[B] \leq(c(r+1) \log (r+1))^{g+1} .
$$

Hence, Theorem 3 follows by induction.

Remarks. (i) The proof of Theorem 3 given above is essentially that of Popken [15], while that of Theorem 2 above is different and much simpler. The measure established by Popken was of the form $\left.c g \log g(r+2) \log ^{2}(r+2)\right)^{-g}$, which is much worse.

(ii) Clearly, Theorem 3 can also be derived under a weaker hypothesis that $f(p) \neq 0$ for infinitely many primes $p$.

A slightly different analysis of the proof of Theorem 3 leads to the following qualitative result.

Theorem 4. Let $D(s)=\sum f(n) / n^{s}$ be in $\mathscr{D}$. If $D$ is differentially algebraic, then those $n$ for which $f(n) \neq 0$ contain only finitely many prime factors.

Proof. Using the same terminology as in the proofs of Theorems 2 and 3, we see that

$$
0=F(p m) v(p m)=f(p) \sum_{j=0}^{r} a_{j}(m)(\log p)^{j} .
$$


Since the polynomial factor on the right-hand side has only finitely many zeros, then for all primes sufficiently large, say $p \geq p_{0}$, we get $f(p)=0$. Furthermore, by the results of Theorem 2 , for $p \geq p_{0}$, we have

$$
\begin{aligned}
0 & =F(p \cdot 2 m) v(p \cdot 2 m)=\sum_{j=0}^{r} \sum_{k \mid 2 m} f(p k) v(p k)(-\log p k)^{j} F_{j}(2 m / k) \\
& =f(2 p) v(2 p) \sum_{j=0}^{r}(-\log 2 p)^{j} F_{j}(m),
\end{aligned}
$$

using the defining property of $m$ and the fact that $f(p)=0$. Thus

$$
f(2 p)=0 \quad\left(p \geq p_{0}\right) .
$$

In general, for each positive integer $k$ and all primes $p \geq p_{0}$, we have by induction that $f(k p)=0$, which implies the assertion of the theorem.

An interesting corollary to Theorem 4 is the following result, whose weaker form was first given by Popken [15].

Theorem 5. Let $D(s)$ be a Dirichlet series and $\zeta(s)$ be the Riemann zeta function. If $D$ satisfies an algebraic differential equation with complex coefficients of order $r$ and total degree $g$, then

$$
|\zeta-D| \geq \begin{cases}(c(r+1) \log (r+1))^{-g} & \text { if } r \geq 1 \\ 2^{-g} & \text { if } r=0\end{cases}
$$

where $c$ is a positive, absolute, and computable constant.

Proof. Since $D$ formally satisfies $P\left(D, D^{\prime}, \ldots, D^{(r)}\right)=0$, where $P$ is a nontrivial polynomial with complex coefficients of total degree $g$. Using the Taylor expansion for polynomial, we get

$$
\begin{aligned}
P\left(\zeta, \zeta^{\prime}, \ldots, \zeta^{(r)}\right)= & \sum_{j=0}^{r}\left(\zeta^{(j)}-D^{(j)}\right) P_{j}\left(D, D^{\prime}, \ldots, D^{(r)}\right) \\
& +\sum_{\substack{0 \leq j \leq k \leq r\\
}}\left(\zeta^{(j)}-D^{(j)}\right)\left(\zeta^{(k)}-D^{(k)}\right) P_{j, k}\left(D, D^{\prime}, \ldots, D^{(r)}\right) \\
& + \text { higher order terms, }
\end{aligned}
$$

where $P_{j}, P_{j, k}, \ldots$ denote the terms connected with higher order partial derivatives of $P$. Observe that for $0 \leq i, j \leq r$ we have

$$
\begin{gathered}
0=\left|\zeta^{(j)}-D^{(j)}\right| \leq|\zeta-D| \leq 1, \\
\left|P_{j}\left(D, D^{\prime}, \ldots, D^{(r)}\right)\right| \leq 1, \quad\left|P_{j, k}\left(D, D^{\prime}, \ldots, D^{(r)}\right)\right| \leq 1, \ldots
\end{gathered}
$$

Applying Theorem 3 and the non-archimedean property of the valuation, we have the desired result.

Our final result gives a measure for differential-difference transcendence of the Riemann zeta function. Since the proof runs in a manner similar to that of Theorem 3, we merely sketch it. 
Theorem 6. Let $Q\left(X_{11}, \ldots, X_{1 J}, \ldots, X_{I 1}, \ldots, X_{I J}\right)$ be a nontrivial polynomial of total degree $g$ with complex coefficients. Let $t_{1}<t_{2}<\cdots<t_{I}$ be an increasing sequence of I real numbers and $0 \leq r_{1}<r_{2}<\cdots<r_{J}=r$ be an increasing sequence of $J$ nonnegative integers. Put $\zeta_{i j}(s)=\zeta^{\left(r_{j}\right)}\left(s-t_{i}\right)$ and $Q(\underline{\zeta})=Q\left(\zeta_{11}, \ldots, \zeta_{1 J}, \ldots, \zeta_{11}, \ldots, \zeta_{I J}\right)$. Then

$$
|Q(\underline{\zeta})| \geq\{(r+1) I\}^{-g}\{c \log (r+1) I\}^{-g+1},
$$

where $c$ is a positive, absolute, computable constant.

Proof. If $\operatorname{deg} Q=0$ then $|Q(\underline{\zeta})|=1$. If $\operatorname{deg} Q=1$, then as in the proof of Theorem 3, the coefficients of $Q(\underline{\zeta})$, considered as a Dirichlet series, take the form

$$
\sum_{i, j} a_{i j} n^{t_{i}}(-\log n)^{r_{j}}
$$

This is an exponential polynomial with real and distinct frequencies $t_{i}$ and with nontrivial polynomial coefficients. By a well-known theorem (see, e.g., Gelfond and Linnik [5, Lemma 1, Chapter 12] or Pólya-Szegö [10, Problem 15, p. 46]), this exponential polynomial has at most $(r+1) I-1$ real zeros, and this gives the theorem when $g=1$. The general case follows by induction in the same manner as that of Theorem 3 .

\section{REFERENCES}

1. T. M. Apostol, Introduction to analytic number theory, Springer-Verlag, New York, 1976.

2. R. Bellman and H. N. Shapiro, The algebraic independence of arithmetic functions (I) multiplicative functions, Duke Math. J. 15 (1948), 229-235.

3. L. Carlitz, Independence of arithmetic functions, Duke Math. J. 19 (1952), 65-70.

4. E. D. Cashwell and C. J. Everett, The ring of number-theoretic functions, Pacific J. Math. 9 (1959), 975-985.

5. A. O. Gelfond and Yu. V. Linnik, Elementary methods in the analytic theory of numbers, Pergamon Press, Oxford, 1966.

6. V. Laohakosol, K. Kongsakorn, and U. Leerawat, Some arithmetic functions algebraically independent with respect to convolution, Soochow J. Math. 15 (1989), 171-178.

7. __ Algebraic independence test of arithmetic functions using Jacobians, J. Sci. Soc. Thailand 15 (1989), 133-138.

8. P. J. McCarthy, Introduction to arithmetic functions, Springer-Verlag, New York, 1986.

9. A. Ostrowski, Über Dirichletsche Reihen und algebraische differentialgleichungen, Math $\mathrm{Z}$. 8 (1920), 241-298.

10. G. Pólya and G. Szegö, Problems and theorems in analysis, vol. II, Springer-Verlag, Berlin, 1976.

11. J. Popken, Algebraic dependence of arithmetic functions, Proc. Kon. Ned. Akad. Wetensch. 65 (1962), 155-168.

12. __, On multiplicative arithmetic functions, Studies in Math. Analysis and Related Topics: Essays in Honour of G. Polya, Stanford Univ. Press, 1962, pp. 285-293.

13. Note on a generalization of a problem of Hilbert, Proc. Kon. Ned. Akad. Wetensch. 68 (1965), 178-181.

14. __ Algebraic independence of certain zeta functions, Proc. Kon. Ned. Akad. Wetensch. 69 (1966), 1-5.

15. __ A measure for the differential-transcendence of the zeta-function of Riemann, Number Theory and Analysis, Papers in Honour of Edmund Landau, Plenum Press, New York, 1969, pp. 245-255. 
16. H. N. Shapiro, On the convolution ring of arithmetic functions, Comm. Pure Appl. Math. 25 (1972), 187-336.

17. H. N. Shapiro and G. H. Sparer, On algebraic independence of Dirichlet series, Comm. Pure Appl. Math. 39 (1986), 695-745.

18. L. I. Wade, Algebraic independence of certain arithmetic functions, Duke Math. J. 15 (1948), 237.

Department of Mathematics, Kasetsart University, BangkoK 10900, Thailand 